Gynäkologische Endokrinologie 2011 · 9:7-7 DOI 10.1007/s10304-010-0385-7

Online publiziert: 15. Januar 2011

(c) Springer-Verlag 2011

\author{
T. Strowitzki ${ }^{1}$ W. Küpker ${ }^{2}$ \\ ${ }^{1}$ Abteilung für Gynäkologische Endokrinologie und Fertilitätsstörungen, \\ Universitäts-Frauenklinik, Heidelberg \\ ${ }^{2}$ Endometriosezentrum, Stadtklinik Baden-Baden
}

\title{
Hormone und Psyche
}

Die Wechselbeziehungen zwischen Hormonen und Psyche sind vielschichtig. Jeder von uns konnte die Auswirkungen in der täglichen Praxis schon häufig beobachten - sei es als zyklusabhängig veränderte Stimmungslage oder in Form von anderen bekannten Phänomenen, z. B. depressiven Phasen im Wochenbett, die eindeutig mit Hormonen zusammenhängen. Auch psychiatrische Erkrankungen wie etwa die Demenz scheinen hormonal beeinflussbar zu sein. Dies spiegelt sich wieder in einer großen Zahl an Studien, welche die Hormonersatztherapie und deren Einfluss auf die Demenz untersuchen.

In der vorliegenden Ausgabe von $G y$ näkologische Endokrinologie wollen wir einige Aspekte der Wechselwirkung zwischen Hormonen und Psyche näher betrachten. Hierfür konnten wir führende Wissenschaftler als Autoren gewinnen.

\section{จ Auch im Gehirn werden Östrogene gebildet}

Dr. Janine Prange-Kiel, Professorin in Dallas, wird sich in ihrem Beitrag mit den Grundlagen der Forschung über Östrogenwirkungen im Gehirn befassen. Auch wenn schon lange bekannt, so ist es doch noch lange kein Allgemeingut, dass auch im Gehirn Östrogene gebildet werden.

Dr. Petra Stute aus Bern hat sich des wichtigen Themas angenommen, wie Hormone und Gehirnfunktionen im Alterungsprozess des Menschen zusammenspielen. Frauen erkranken im Vergleich zu Männern doppelt so häufig an einer Demenz, sodass hormonale Unterschiede hier durchaus eine Rolle spielen könnten.

Diesem Thema widmen sich auch die beiden Psychiater Prof. Peter Schön- knecht aus Leipzig und Prof. Johannes Schröder aus Heidelberg. In ihrem Beitrag beleuchten sie die Zusammenhänge zwischen Hormonen und der AlzheimerDemenz und diskutieren die potenziellen präventiven Therapieeffekte, die sich gegenwärtig daraus ableiten lassen.

Der Psychiater Dr. Niels Bergemann beschäftigte sich schon in seiner Heidelberger Zeit ausführlich mit den Zusammenhängen zwischen schizophrenen Psychosen und Östrogenen. Er zeigt in seinem Artikel nicht nur die vulnerablen Lebensphasen für Östrogenmangelzustände auf, sondern stellt den aktuellen Erkenntnisstand darüber dar, ob und in welchem Umfang Östrogene in der antipsychotischen Therapie eingesetzt werden können.

Auch die Hormontherapie selbst nimmt Einfluss auf die Psyche. In diesem Zusammenhang werden immer wieder die Auswirkungen der hormonalen Kontrazeption mit der Antibabypille auf das psychische Wohlbefinden thematisiert. Was daran ist, wird Prof. Johannes Bitzer aus Basel näher erläutern.

\section{Die Bezeichnung "prosoziales Hormon" für Oxytocin ist durchaus berechtigt}

Zuletzt haben wir in dieser Ausgabe für Sie noch ein besonderes Thema aufbereitet. Die Psychiaterin Prof. Sabine Herpertz aus Heidelberg ist die ausgewiesene Expertin und Forscherin über die Wirkungen von Oxytocin und verdeutlicht in ihrem Beitrag, dass die Bezeichnung „prosoziales Hormon“ für Oxytocin durchaus berechtigt ist - eine für uns als Gynäkologen sehr interessante Betrachtungsweise.
Mit dem vorliegenden Heft haben wir sicherlich einige interessante und neue Aspekte über Hormone und Psyche für Sie zusammengestellt. Wichtig war uns vor allem, den Blickwinkel anderer Fachrichtungen einzubeziehen. Bei der Vorbereitung hatten wir viel Spaß und konnten unseren Wissensstand deutlich erweitern. Nun hoffen wir sehr, dass Sie nach der Lektüre genauso empfinden.

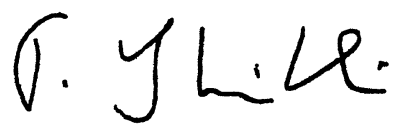

Prof. Dr. Thomas Strowitzki

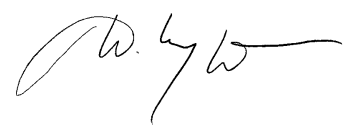

Prof. Dr. Wolfgang Küpker

\section{Korrespondenzadresse \\ Prof. Dr. T. Strowitzki}

Abteilung für Gynäkologische Endokrinologie und Fertilitätsstörungen,

Universitäts-Frauenklinik

Voßstr. 9, 69115 Heidelberg

thomas.strowitzki@med.uni-heidelberg.de 